\title{
$\mathrm{N}$-糖链輴液酸连接异构体的质谱分析方法研究进展
}

\author{
李月悦 $a$ 彭叶 $a$ 陆豪杰*, $a, b$ \\ ( ${ }^{a}$ 复旦大学生物医学研究院和化学系 上海 200032) \\ ( $b$ 卫健委糖复合物重点实验室(复旦大学) 上海 200032)
}

\begin{abstract}
摘要 蛋白质在翻译过程中、翻译过程后会发生糖基化. 糖基化会以直接或间接的方式影响蛋白质的功能及其相互作 用, 并与多种人类疾病有关, 其中, 唾液酸化 $\mathrm{N}$-糖链在一些重要的生理和病理过程中发挥关键作用. 已知的唾液酸与 相邻单糖之间的连接方式包括 $\alpha-2,3-、 \alpha-2,6-、 \alpha-2,8-、 \alpha-2,9-$ 连接, 连接方式不同的唾液酸化 N-糖链在细胞活动、生命 体的生理和病理过程中的功能往往不同. 质谱技术是分析 N-糖链的重要工具, 它能够快速和灵敏地检测 N-糖链, 通过 将色谱技术以及衍生化方法等与质谱联用可以实现对唾液酸化 N-糖链及其连接异构体的分离和检测. 本文主要围绕 $\alpha$-2,3-和 $\alpha$-2,6-连接的唾液酸化 $\mathrm{N}$-糖链进行综述, 介绍它们的结构和在细胞活动及疾病中不同的功能, 并综述近年来基 于质谱的唾液酸化 $\mathrm{N}$-糖链的连接异构体分析方法以及这些方法在生物医学领域的应用, 并对未来的生物医学研究提 供新的思路和途径.
\end{abstract}

关键词 N-糖链; 唾液酸; 质谱; 衍生化; 异构体分离

\section{Advances in Analysis of Linkage Isomers of Sialylated N-Glycans by Mass Spectrometry}

\author{
Yueyue $\mathrm{Li}^{a} \quad$ Ye Peng ${ }^{a} \quad$ Haojie $\mathrm{Lu}^{*, a, b}$
}

( ${ }^{a}$ Institutes of Biomedical Sciences and Department of Chemistry, Fudan University, Shanghai 200032, China) $\left({ }^{b}\right.$ NHC Key Laboratory of Glycoconjugates Research, Fudan University, Shanghai 200032, China)

\begin{abstract}
Protein glycosylation occurs during and after the translation process, and glycosylation will diversify the function of the protein. Glycosylation directly or indirectly affects the function of proteins and their interactions, and is related to a variety of human diseases. Among them, sialylated N-glycans play a key role in many important physiological and pathological processes. Sialic acid usually bonds with adjacent monosaccharides via $\alpha-2,3-, \alpha-2,6-, \alpha-2,8-$ or $\alpha-2,9-$ linkage. Sialylated $\mathrm{N}$-glycans with different linkage always have different functions in cell activities, physiological and pathological processes of living organisms. Mass spectrometry is an important tool for the analysis of N-glycans. It can quickly and sensitively detect N-glycans. The separation and detection of sialylated N-glycans and their linked isomers can be achieved by combining chromatographic techniques and derivatization methods with mass spectrometry. In this review, we mainly focuses on $\alpha-2,3-$ and $\alpha$-2,6-linked sialylated $\mathrm{N}$-glycans, introduces their structure and different functions in cell activities and various diseases, and summarizes the analysis methods of linked isomers of sialylated $\mathrm{N}$-glycans based on mass spectrometry and the application of these methods in the field of biomedicine in recent years. This review can provide new ideas and approaches for future biomedical research.
\end{abstract}

Keywords N-glycan; sialic acid; mass spectrometry; derivatization; isomeric separation

\section{$1 \mathrm{~N}-$ 糖链和唾液酸化 $\mathrm{N}-$ 糖链的结构与生物学 功能}

\section{$1.1 \mathrm{~N}$-糖链的结构与生物学功能}

糖基化是在糖基转移酶的非模板竞争催化下, 糖链 连接到蛋白质或者脂质上形成糖复合物的过程. 根据糖 链与蛋白质的连接方式, 蛋白质的糖基化主要分为三种 类型: $\mathrm{N}$-糖基化、 $\mathrm{O}$-糖基化、 $\mathrm{C}$-糖基化. Apweiler 等 ${ }^{[1]}$ 通
过分析 SWISS-PROT 数据库中已知蛋白质的糖基化位 点发现 $84 \%$ 的糖蛋白中有 $\mathrm{N}-$ 糖基化的位点.

$\mathrm{N}$ - 糖基化的过程需要识别特征氨基酸序列 Asn-X-Ser/Thr/Cys (Asn 是天冬酰胺, $\mathrm{X}$ 是除脯氨酸 Pro 以外的任何一个氨基酸, Ser 是丝氨酸, Thr 是苏氨酸, Cys 半胱氨酸, 其中 Thr 比 Ser 更常见, Cys 最少见 ${ }^{[2]}$ ). N糖链与蛋白质的天冬酰胺侧链的酰胺氮原子结合. $\mathrm{N}$-糖 链中的单糖有唾液酸(sialic acid 或 N-acetylneuraminic acid, SA 或 NeuAc)、半乳糖(galactose, Gal)、甘露糖

*E-mail: luhaojie@fudan.edu.cn; Tel.: 021-54237618

Received February 6, 2021; published March 29, 2021.

Project supported by the National Key Research and Development Program of China (No. 2016YFA0501303) and the National Natural Science Foundation of China (No. 21974025).

项目受国家重点研发项目(No. 2016YFA0501303)和国家自然科学基金(No. 21974025)资助. 
(mannose, Man)、岩藻糖(fucose, Fuc)、N-乙酰葡萄糖胺 (N-acetylglucosamine, GlcNAc) 等, 糖链中通常包含几个 到几十个单糖. 单糖组成的多样性、单糖单元间连接方 式的不同(如唾液酸的 $\alpha-2,3-/ \alpha-2,6$-连接)、分支结构等都 会造成 $\mathrm{N}$-糖链结构的多样. 蛋白质的糖基化具有宏观 不均一性和微观不均一性, 宏观不均一性是指一个蛋白 质可糖基化不同位点和不同修饰程度; 微观不均一性是 指一个糖基化位点上连接有多种不同结构的糖链 ${ }^{[3]}$.

$\mathrm{N}$-糖链的类型有高甘露糖型、复杂型、杂合型, 由 不同的糖基转移酶催化形成. 高甘露糖型糖链的分支中 只有甘露糖; 复杂型糖链有乙酰葡萄糖胺、唾液酸等按 照一定顺序连接的分支; 杂合型糖链中包含高甘露糖型 和复杂型两种分支(图 1). N-糖链还可以根据其五糖核 心外的延伸类型分为二分支 (bi-antennary), 三分支 (tri-antennary)和四分支(tetra-antennary)结构.

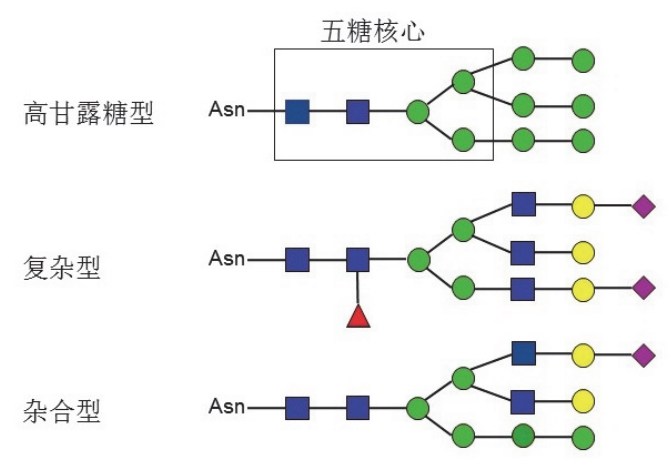

$\square$ 乙酰半乳糖胺（N-acetylglucosamine, GalNAc)
岩藻糖 (fucose, Fuc) $\bigcirc$ 半乳糖 (galactose, Gal)
$\checkmark$ 唾液酸 (sialic acid, SA) $\Delta$ 岩藻糖 (fucose, Fuc)

图 1 N-糖链的种类和结构示意图

Figure 1 Schematic diagram of the types and structure of N-glycans

$\mathrm{N}$-糖链参与许多重要的生命过程, 如影响细胞间信 号传导以及识别粘附等过程 ${ }^{[4-5]}$. N-糖链还可以在细胞 内吞的过程中参与信号调节过程, 当某种 $\mathrm{N}-$ 糖链与细 胞表面的凝集素识别并结合时, 细胞便停止内吞连接该 种 $\mathrm{N}$-糖链的糖蛋白, 该过程可以改变细胞表面糖蛋白 的丰度. N-糖链在免疫细胞的分化和激活过程中也会发 生较大变化, 从而影响免疫细胞的抗原识别和免疫系统 稳态维持等过程. 例如, GlcNAC $\beta$-1,6-连接的 N-糖链分 支可以影响 $\mathrm{T}$ 细胞受体的信号传递, 研究表明半乳糖凝 集素(Galectin)和细胞表面的 $\mathrm{T}$ 细胞受体的相互作用依 赖于合成 GlcNAC $\beta-1,6$-连接的 $\mathrm{N}$-糖链分支的糖基转移 酶 V (Mgat5)的存在, 在有超敏反应的延迟型小鼠模型 中, 若合成该糖链分支的 Mgat5 缺失, 会引发自身免疫 性脑脊髓炎 ${ }^{[6]}$.

在 20 世纪 60 年代人们发现了癌的扩散与人体内糖 基化水平改变之间的联系. 造成癌细胞糖基化表达变化
的因素有糖基转移酶过度表达或表达水平下调，糖基转 移酶在高尔基体的定位变化和新生成的糖肽三维结构 或序列的改变等 ${ }^{[7]}$. 有研究者提出癌症糖基化变化的两 种假说: 不完全合成过程和新合成过程; 不完全合成过 程是指在癌症发生的早期, 由于细胞受到了基因突变或 外界刺激的损害, 糖链的合成被中断, 使得被截短的糖 链表达在新合成的蛋白上; 新合成过程是指在癌症发展 的中后期, 癌细胞的表达具有特异性, 会生成复杂的糖 基结构, 例如聚 N-乙酰乳糖胺和癌抗原唾液酸化糖 链 $^{[8]}$. 随着症细胞表面的糖链变化, 癌细胞的粘附作用 也发生改变, 所以癌细胞更容易脱离原来的组织, 扩散 到其他的组织中, 从而在血清中可以检测到差异性表达 的 N-糖链, 所以越来越多的研究将血清 N-糖链作为潜 在的癌血清标志物.

\section{2 唾液酸化 N-糖链的结构和生物学功能}

唾液酸是有 9 个碳的 $\alpha$-酮醛糖酸, 其系统命名为 5氨基-3,5-二脱氧- $D$-甘油- $D$-半乳壬酮糖酸. 唾液酸主要 存在于糖链的非还原端, 在生理环境下带有负电荷. 目 前已经被发现和鉴定的唾液酸分子及其衍生物有五十 几种, 根据取代基团不同, 唾液酸可被分成三类, 包括 $\mathrm{N}$-乙酰神经氨酸(N-acetylneuraminic acid, Neu5Ac)、N着弪乙酰神经氨酸(N-glycolylneuraminic acid, Neu5Gc)和 酮基-脱氧壬酮糖酸(2-keto-3-deoxynononic acid, KDN). 在高等脊椎动物中, 唾液酸主要以 Neu5Ac 和 Neu5Gc 两种类型存在，它们具有不同的生理作用，人体中最常 见的唾液酸为 Neu5Ac ${ }^{[9]}$. 唾液酸可以通过羟基以 $\alpha-2,3-$ 或 $\alpha-2,6$-形式连接半乳糖、N-乙酰半乳糖胺和 N-乙酰葡 萄糖胺, 还可以通过 $\alpha-2,8$-或 $\alpha-2,9$-形式连接多个唾液 酸.

连接有唾液酸的糖链主要分布在哺乳动物细胞表 面或一些分泌蛋白质表面，在细胞表面粘附或信号识别 中起着重要的作用. 例如, 唾液酸与半乳糖之间主要以 $\alpha$-2,3-和 $\alpha$-2,6-两种方式连接，禽流感病毒趋向于结合 $\alpha-2,3$-连接的唾液酸化 $\mathrm{N}$-糖链, 所以人类上皮细胞表面 广泛表达的 $\alpha-2,6$-连接唾液酸化 $\mathrm{N}$-糖链可以保护人类免 受感染, 而禽流感 $\mathrm{A}$ 型病毒, 例如 H5N1 和 H9N2, 能从 鸟类传给人类, 是因其识别模式从识别 $\alpha-2,3-$-连接的唾 液酸转变成识别 $\alpha-2,6$-连接的唾液酸 ${ }^{[10]}$.

唾液酸化修饰屏蔽了与之相连的糖蛋白及糖脂结 构, 稳定细胞膜结构, 保护细胞和大分子不受酶及免疫 系统的攻击, Zlatina 等[11]发现, 在免疫系统形成中性粒 细胞胞外陷阱(neutrophil extracellular traps, NETs)捕获 病原体时会形成高浓度的胞外组蛋白，从而产生细胞毒 性, 但聚唾液酸(polysialic acid, polySia)糖链会结合具有 细胞毒性的组蛋白, 降低其细胞毒性. Lv 等 ${ }^{[12]}$ 通过对比 分析发现, 相对于正常人, 肝癌患者血清的囊泡中有 24 种 $\mathrm{N}$-糖链的相对丰度增加, 其中一半以上包含岩藻糖 或唾液酸. 
有研究发现癌细胞表面与正常细胞的不同主要在 于唾液酸的变化 ${ }^{[13-14]}$. 唾液酸水平、连接方式和分布的 变化与癌症恶性转化的各个方面相关, 其中细胞表面唾 液酸水平的提高会增强癌细胞的转移潜能 ${ }^{[15-16]}$. 在女性 中, 乳腺癌是最常见的癌症 ${ }^{[17]}$, 并是仅次于肺癌的第二 大常见转移性癌症, 约 $10 \% \sim 16 \%$ 乳腺癌患者的癌细胞 会向脑部转移 ${ }^{[18]}$. Peng 等 ${ }^{[19]}$ 联用液相色谱(liquid chromatography, LC)和串级质谱(tandem mass spectrometry, $\mathrm{MS} / \mathrm{MS}$ )对比了 5 个乳腺癌细胞系和 1 个脑癌细胞系中 $\mathrm{N}$-糖链唾液酸连接异构体的表达, 发现 $\alpha-2,6$-连接的唾 液酸化 $\mathrm{N}$-糖链的增加有望作为乳腺癌脑转移的指标. 另外, 在癌症细胞中, $\beta-1,6-G 1 c N A c$ 分支酶, N-乙酰氨基 葡萄糖转移酶 $\mathrm{V}$ (N-acetylglucosaminyl transferase $\mathrm{V})$ 的 活性增加, 会导致 $\mathrm{N}$-糖链的分支数量增加, 从而为糖链 末端唾液酸化提供了更多的位点; 唾液酸转移酶 (sialyltransferase)表达增加会提高细胞表面唾液酸化程 度, 从而促进癌细胞的转移 ${ }^{[20]}$. 唾液酸化程度的增加还 表现在 polySia 的表达, polySia 是一种与多种癌症, 例如 神经母细胞瘤(neuroblastoma)、非小细胞肺癌(non-small cell lung carcinoma)、乳腺癌(breast cancer)等相关的癌胚 抗原(oncofetal antigen) ${ }^{[21]}$.

唾液酸化 $\mathrm{N}$-糖链在脑中的含量很高, 其可以促进 神经细胞的分化、发育和再生等, Galuska 等 ${ }^{[22]}$ 发现参与 神经系统发育的神经细胞粘附分子(neural cell adhesion molecule, NCAM) 可以形成特殊的聚唾液酸化结构修饰, 该修饰带较多负电荷, 会影响细胞表面粘附作用及细胞 间通讯过程, 例如神经元祖细胞(neuronal progenitor cells)的迁移, 从而影响神经系统的发育.

此外有研究者发现血浆中游离的唾液酸和唾液酸 化糖链可以作为疾病的潜在标志物, Ferrer-Batalle 等[23-24]发现高危前列腺癌患者前列腺液中前列腺特异 性抗原(prostate specific antigen, PSA)的 $\alpha-2,3$-唾液酸的 百分比明显高于中低危前列腺癌症患者和前列腺增生 患者, 他们提出将该百分比和前列腺健康指数(prostate health index, PHI) 结合检测前列腺癌症, 灵敏度为 $100 \%$, 特异度为 $94 \%$, 这一组合极大提高了前列腺癌检 测的准确性, 可以有效区分前列腺癌患者和良性前列腺 增生患者, 证明了 $\alpha-2,3$-唾液酸用于鉴别高危前列腺癌 的潜力. 肺癌是最常见的癌症 (占总癌症病例数的 $11.6 \%$ )之一, 也是癌症中死亡人数最多的(占总癌症死 亡的 $18.4 \%)^{[17]}$. Vasseur 等 ${ }^{[25]}$ 通过识别性衍生化方法和 基质辅助激光解吸电离质谱(matrix assisted laser desorption/ionization mass spectrometry, MALDI-MS)检测发现, 相对于健康人血清, 肺癌患者的血清中含有三个和两个 $\alpha-2,3$-连接唾液酸的 $\mathrm{N}$-糖链异构体含量降低, 含有两个 $\alpha-2,6$-连接唾液酸的 $\mathrm{N}$-糖链异构体含量升高. 卵巢癌是 造成美国女性癌症死亡的五大癌症类型之一[26], 早期 诊断对卵巢癌患者的长期存活率至关重要, 卵巢癌常规 肿瘤标志物 CA125 的特异性为 94\% 98.5\%, 但是其敏
感度较低，目前只有约 $20 \% \sim 25 \%$ 的卵巢癌患者能得到 早期诊断 ${ }^{[27]}$. Dedova 等 ${ }^{[28]}$ 通过识别性衍生化方法和 MALDI-TOF-MS 检测发现，早、晚期卵巢癌患者 $\alpha-2,3-/ \alpha-2,6$-连接的唾液酸比值显著高于健康人对照组, 他们认为该比值升高能用来检测早、晚期卵巢癌. 在前 列腺癌患者 ${ }^{[29]}$ 和恶性胶质瘤患者 ${ }^{[30]}$ 血清中 $\alpha-2,3$-连接的 唾液酸化水平升高; 另外, 肿瘤细胞中 $\alpha-2,6$-唾液酸水 平升高, 主要是因为 $\alpha-2,6$ - 连接唾液酸转移酶 ST6Gal-I ${ }^{[31-33]}$ 或 ST6GalNAc ${ }^{[34-35]}$ 上调.

\section{2 唾液酸化 $\mathrm{N}$-糖链质谱分析的主要目的和连 接异构分析的挑战}

近年来，质谱分析技术在糖链研究领域得到很多应 用, 串级质谱(tandem mass spectrometry, MS/MS)的发展 为糖链序列和精细结构的解析提供了更好的技术平 台 ${ }^{[36-37]}$, 在唾液酸糖链的分析领域也发挥着重要的作 用. 生物质谱对糖链的检测限已达到 fmol 至 amol 水平, 检测的动态范围为 $4 \sim 5$ 个数量级 ${ }^{[38]}$. 质谱对唾液酸化 $\mathrm{N}$-糖链进行的分析可以得到唾液酸的数目、连接方式等 结构和相对含量等信息, 有利于分析唾液酸化 N-糖链 在生理病理方面的作用.

但质谱在分析唾液酸糖链时也存在一定困难, 唾液 酸与 $\mathrm{N}$-糖链连接的键键能低, 在高能质谱离子化情况 下发生断裂容易造成唾液酸丢失; 唾液酸糖链上带有羧 基，在质谱正离子模式下难以使质子带正电，导致分析 灵敏度低; 此外, 多个唾液酸残基在正离子模式分析时, 会产生阳离子加合物, 导致质谱图复杂难解析. 特别是 连接方式不同的唾液酸糖链在质谱图中的质荷比 $(m / z$ 值)相同, 难以进行识别, 因此, 研究人员发展了基于质 谱的非衍生和衍生的多种方法来检测唾液酸糖链异构 体.

\section{3 基于质谱的 $\mathrm{N}$-糖链唾液酸化连接异构体的 研究方法}

质谱检测唾液酸化 N-糖链时, 非衍生法和衍生法 都能进行有效的 $\mathrm{N}$-糖链唾液酸连接异构体分析. 非衍 生方法通常是利用色谱或者电泳方法依据 $\alpha$-2,3-和 $\alpha-2,6$-连接的唾液酸之间的性质差异进行分离, 还可以 用离子淌度质谱(ion mobility mass spectrometry, IMMS) 等根据结构差异进行分离. 衍生方法通常利用 $\alpha-2,3$-和 $\alpha-2,6$-连接的唾液酸之间的羧基反应活性差异, 在合适 的反应条件下， $\alpha-2,3$-连接唾液酸的羧基倾向于形成内 酯，而 $\alpha-2,6$-连接的唾液酸倾向于与其他亲核试剂反应. 通过优化反应条件, $\alpha-2,6$-和 $\alpha-2,3$ 连接的唾液酸可以选 择性地转化为不同的分子，在这些连接异构体之间产生 质量差异，从而可以用质谱进行识别检测.

相较于衍生化方法, 非衍生方法的样品不需要进行 复杂的前处理, 可以直接进行相应的表征, 但是其仅依 
赖于 $\alpha$-2,3-和 $\alpha$-2,6-连接的唾液酸之间的性质差异进行 分离, 分离条件较为严格, 分离的选择性也往往受到限 制. 衍生化方法的目的一方面是为了提高 $\mathrm{N}$-糖链在质 谱中的离子化效率和避免唾液酸的源内丢失, 另一方面 是通过衍生实现异构体更高选择性的分离, 并进行较为 准确的定量, 但同时, 用于衍生化方法的样品需要较为 复杂的衍生化步骤, 容易造成样品的丢失.

\section{1 唾液酸化 N-糖链的非衍生质谱分析方法}

唾液酸化 $\mathrm{N}$-糖链的非衍生质谱分析方法通常会将 高效阴离子交换色谱(high performance anion-exchange chromatography, HPAEC)或多孔石墨化碳(porous graphitized carbon, PGC)柱等分离 $\mathrm{N}$-糖链的方法与质谱相结 合, 也有研究者采用毛细管电泳(capillary electrophoresis, CE) 和质谱联用的方法以及离子淌度质谱的方法进 行分析.

\section{1 .1 基于 HPAEC 的分析方法}

糖类分子中有较多的羟基, 在强碱溶液中可以解离 成阴离子, 所以在高 $\mathrm{pH}$ 值的流动相中其部分或全部以 阴离子形式存在, 可以通过控制流动相的 $\mathrm{pH}$ 值调节糖 链中羟基的解离, 从而根据其在阴离子交换柱上的保留 不同而得到分离, 同时唾液酸 $\mathrm{N}$-糖链中的羧基也能够 进行解离, 从而被 HPAEC 分离 ${ }^{[39]}$. 对于唾液酸化 N-糖 链, 唾液酸数目增多, 糖链的酸性增加, 所以根据糖链 在 HPAEC 中的洗脱顺序不同可以对含不同数目唾液酸 的糖链进行分离 ${ }^{[40]}$. Zamze 等 ${ }^{[41]}$ 通过弱阴离子交换色谱 分离大鼠脑组织样品中用糖苷酶 F (PNGase F) 释放的糖 链, 他们根据单、双、三和四唾液酸化糖链洗脱时间不 同，分出几个主要洗脱时间区域. HPAEC 能根据样品的 电荷、大小、组成和连接方式来分离糖链, 并且结合其 他分离模式和分离技术可以实现二维分离. Bones 等 ${ }^{[42]}$ 用酶解释放生物参考制剂 3 促红细胞生成素标准品 (biological reference preparation 3 erythropoietin standard) 中的 $\mathrm{N}$-糖链后, 将阴离子交换柱作为基于唾液酸数目 和糖链大小的第一维分离, 亲水相互作用色谱 (hydrophilic interaction chromatography, HILIC)作为第二 维分离, 随后用质谱检测, 获得糖链结构信息, 样品中 最丰富的是有多个唾液酸和多个聚 N-乙酰乳糖胺的四 分支糖链.

HPAEC 能分离唾液酸化 N-糖链的连接异构体, 是 由于两种异构体中参与交换的羟基数目不同. $\alpha-2,6-$-连 接的唾液酸化糖链在高 $\mathrm{pH}$ 阴离子交换色谱中的保留时 间比 $\alpha-2,3$-连接的唾液酸化糖链短, 这可能是因为 $\alpha-2,6$-连接的唾液酸化糖链中的 $6-\mathrm{OH}$ 未参与色谱柱中 的交换, 参与交换的电荷总数减少 ${ }^{[43]}$. Maier 等 ${ }^{[44]}$ 用 HPAEC-MS/MS 分析人血浆免疫球蛋白 G (immunoglobulin G, IgG) 中的 N-糖链时, 检测到 4 个唾液酸化 N糖链的连接异构体.

HPAEC 可以较为高效地分离 $\mathrm{N}$-糖链唾液酸连接异
构体，并且 HPAEC 的色谱柱具有 $\mathrm{pH}$ 范围广、容易再生 和使用寿命长的优点, 在结合质谱等分析手段之后还可 以进行 $\mathrm{N}-$ 糖链的结构分析.

\section{1 .2 基于 PGC 柱的分析方法}

PGC 柱是分离糖链的有力工具, 在糖链分离领域 应用比较广泛. PGC 对天然糖链的保留较强, 并能保留 小分子糖链 ${ }^{[45]}$, 可用于分离衍生化或非衍生化的 N-糖 链 ${ }^{[46]}$. PGC 的高效分离性能是基于疏水相互作用和极性 或静电相互作用, 此外因固定相分子是平面性的, 被分 析物的三维结构也影响保留时间 ${ }^{[47]}$, 因此 PGC 可以用 来分离 $\mathrm{N}$-糖链唾液酸连接异构体 ${ }^{[48]}$.

Pabst 等 ${ }^{[49]}$ 联用 PGC 分离方法与电喷雾电离质谱 (electrospray ionization mass spectrometry, ESI-MS), 分 析了多种动物的纤维蛋白原(fibrinogen)酶解释放的糖 链结构, 结果显示在弱酸性条件下 PGC 色谱柱可以分 离单唾液酸化和二唾液酸化 $\mathrm{N}$-糖链各种可能的异构体, 以及 $\alpha-2,3$-和 $\alpha-2,6$-连接的唾液酸所有可能的异构体组 合. Ashwood 等[50]利用 PGC-LC-ESI-MS/MS, 根据得到 的谱图中两个以上高特异性离子片段来鉴别 $\mathrm{N}$-糖链结 构中的唾液酸化结构, 从而分析了牛胎球蛋白和人 $\mathrm{IgG}$ 的 $\mathrm{N}$-糖链的唾液酸连接异构体. Anugraham 等 ${ }^{[51]}$ 用 PNGase F 从健康卵巢表面上皮细胞系(HOSE 6.3 和 HOSE 17.1)和浆液性卵巢癌细胞系(SKOV 3, IGROV1, A2780 和 OVCAR 3)的细胞膜糖蛋白中释放出 N-糖链 后, 用多孔石墨化碳纳米液相色谱和负离子电喷雾电离 质谱对其进行分析，根据其分子量和串级质谱裂解图谱 对糖链结构进行了表征, 异构体分析结果表明在卵巢癌 细胞膜上的 $\alpha-2,6$-连接的唾液酸化 $\mathrm{N}$-糖链的水平升高.

由于 PGC 的分离性能高效, 所以基于 PGC 的分离 方法应用最为广泛，在结合使用质谱等检测方法后可以 在临床中用于 $\mathrm{N}$-糖链唾液酸连接异构体的检测. 虽然 PGC 柱在保留时间的稳定性和重现性上仍然有所欠缺, 但是通过特定试剂洗涤 PGC 柱子可以在一定范围内改 善这一点.

\section{1 .3 基于 $\mathrm{CE}$ 的分析方法}

在电泳分离中被分析物根据在外加电压下的电泳 迁移率不同而被分离. $\alpha-2,3$-和 $\alpha-2,6$-连接的唾液酸化 N糖链 $\mathrm{p} K_{\mathrm{a}}$ 值不同, 分子空间大小不同, 则电泳迁移率不 同，因此能在不进行衍生化的情况下分离 $\mathrm{N}$-糖链唾液 酸连接异构体. Kammeijer 等 ${ }^{[52]}$ 采用 Fuguet 等 ${ }^{[53]}$ 提出的 内标毛细管电泳法, 得到 $\alpha-2,3$-和 $\alpha$-2,6-唾液酸化乳糖 的 $\mathrm{p} K_{\mathrm{a}}$ 值的相对偏差为 $3.4 \times 10^{-2}$, 并应用 CE-ESI-MS 检测了用胰蛋白酶酶解前列腺特异性抗原(prostate specific antigen, PSA) 所得糖肽中的 N-糖链, 鉴定出 75 个 $\mathrm{N}$-糖链, 同时实现了 $\alpha-2,3$-和 $\alpha-2,6$-连接的唾液酸化 $\mathrm{N}$ 糖链的基线分离.

$\mathrm{CE}$ 分析方法发展得相对较早, 成本较低, 设备简 单且分离能力强, 并常常与质谱等其他检测技术结合使 
用, 结合使用后能极大提高 $\mathrm{CE}$ 的分析能力, 在分析 N糖链唾液酸连接异构体时, 能够高选择性地分离异构 体, 同时也为后续利用串联质谱阐明糖链结构提供了机 会.

\section{1 .4 基于 IMMS 的分析方法}

离子通过 IMMS 的管道时, 会受到惰性气体和通常 情况下方向相反的微弱电场作用 ${ }^{[54-55]}$, 离子的电荷, 大 小形状不同，受到的作用力不同，则经过管道的时间不 同, 通过测量离子通过管道的漂移时间, 可转换为碰撞 截面(collision cross section, CCS)值, 能够用于描述结构 和分离糖链异构体.

近年来, 由于离子淌度质谱可以对糖链结构进行表 征, 应用离子淌度质谱分离 $\mathrm{N}$-糖链唾液酸连接异构体 的方法发展迅速, 但是因为结构不同造成的检测数据的 差异相对比较微小, 所以利用离子淌度质谱区分 $\mathrm{N}$-糖 链唾液酸连接异构体时, 通常需要先将糖链碎裂为较小 的碎片, 再选择相应的碎片进行检测, 这是其中较为繁 琐的一步.

Hinneburg 等[56]设计合成了分别带有 $\alpha-2,3-、 \alpha-2,6-$ 单唾液酸化的双分支糖链的糖肽 GP3 和 GP4, 验证 IMMS 对唾液酸异构体的分辨能力. 对于完整的糖肽, 其唾液酸连接方式的不同并不能使它们在 IMMS 中分 离, 但是联用碰撞诱导解离(collision-induced dissociation, CID) 将糖肽碎裂成碎片离子后, $m / z$ 为 657 的 $\mathrm{B}_{3}$ 片 段(Gal、GlcNAc 和 NeuAc 组成的三糖)因 NeuAc 连接 方式的不同表现出明显不同的漂移时间, $\alpha-2,6$-连接的 $\mathrm{B}_{3}$ 离子比 $\alpha-2,3$-连接的 $\mathrm{B}_{3}$ 离子的漂移时间要短. 人血浆 中的糖蛋白 $\alpha-1$ 蛋白酶抑制剂 $(\alpha-1$ proteinase inhibitor, A1PI)主要含有 $\alpha-2,6$-连接的唾液酸残基, 而中国仓鼠卵 巢(chinese hamster ovary, CHO)细胞中重组表达的 A1PI 主要含有 $\alpha-2,3$-连接的唾液酸残基, 他们将这两种细胞 中 A1PI 的混合物进行 CID 碎裂后, 观测 $\mathrm{B}_{3}$ 片段在 IMMS 中漂移时间, 结果得到, 来自 $\mathrm{CHO}$ 和来自人血浆 前体的 A1PI 的 $\mathrm{B}_{3}$ 片段分别与 GP3 和 GP4 得到的 $\mathrm{B}_{3}$ 片 段的漂移时间基本相同, 从而表明 IMMS 可以鉴定 N糖链唾液酸连接异构体.

Barroso 等 ${ }^{[57]}$ 将小鼠转铁蛋白 (mouse transferrin, $\mathrm{mTf}$ )经过胰蛋白酶酶解释放的 N-糖链经过 CID 碎裂后, 联用行波离子迁移谱(traveling wave ion mobility spectrometry, TWIMS)和飞行时间质谱进行分析, 结果显示, $\alpha$-2,6-连接的 H1N1S1 片段比 $\alpha-2,3$-连接的 H1N1S1 片段 的 CCS 值小; 对人类 $\alpha 1$-酸糖蛋白 $(\alpha-1$-acid glycoprotein, $\alpha 1-\mathrm{AGP})$ 糖链的分析也获得了与 mTf 相同的结果; $\alpha 1-A G P$ 经过酶解、碎裂后得到的 H5N4S2 糖链片段, 通 过比较 $\operatorname{CCS}$ 值实现了含两个 $\alpha-2,6$-连接唾液酸的 H5N4S2 片段和含有一个 $\alpha-2,6$-连接唾液酸和一个 $\alpha-2,3-$ 连接唾液酸的 H5N4S2 片段的区分.

近年来研究者们使用高场不对称波形离子淌度质 谱(high-field asymmetric waveform ion mobility mass spectrometry, FAIMS)来分离 $\alpha-2,3$-和 $\alpha$-2,6-唾液酸连接 异构体. Lane 等 ${ }^{[58]}$ 通过调整补偿电压和载气中氮气和甲 醇的比例等条件实现了 H2S1、H1N1S1 和 H5N4F1S2 糖链片段的唾液酸连接异构体的分离.

\section{2 基于衍生的唾液酸化 $\mathrm{N}$-糖链质谱分析方法}

对于唾液酸化 $\mathrm{N}-$ 糖链的中性化, 研究者们最初发 展了全甲基化衍生. 全甲基化是将糖链上游离的羟基变 为甲氧基, 并将唾液酸甲酯化, 同时在 $\mathrm{N}$-乙酰葡萄糖 胺、N-乙酰半乳糖胺和唾液酸的氮原子上加入甲基. 全 甲基化可以中和唾液酸上的负电荷，引进疏水基团，将 质谱的检测灵敏度提高 $10 \sim 20$ 倍, 同时有助于串级质 谱分析，从而鉴别糖链的分支结构 ${ }^{[59]}$. 全甲基化最开始 是使用二甲亚砜( dimethyl sulfoxide, DMSO)作为溶剂, 使用碘甲烷(methyl iodide)来完成反应, 随后研究者们 发现氢氧化钠可以促进反应完全, Novotny 等 ${ }^{[59]}$ 在 DMSO 和碘甲烷混合液中, 利用 $\mathrm{NaOH}$ 固体颗粒辅助衍 生糖链，减少了副反应的发生.

促进全甲基化完全反应需要使用氢氧化钠，但是氢 氧化钠试剂碱性强, 会破坏部分糖链修饰结构, 无法全 面分析糖链 ${ }^{[60]}$, 之后研究者们发展了羧基衍生反应 ${ }^{[61]}$, 酰胺化衍生反应 ${ }^{[62]}$, 乙酰肼衍生 ${ }^{[63]}$ 和对甲基苯胺衍 生 ${ }^{[64]}$ 等一系列温和的衍生反应.

因为 $\alpha-2,3$-连接的唾液酸上的羧基与相邻半乳糖之 间的空间位阻较大，酰胺化和甲酯化不能将其完全衍 生, 但研究者们利用六氟磷酸(7-氮杂苯并三唑-1-氧基) 三吡咯烷磷 ((7-azabenzotriazol-1-yloxy)trispyrrolidinophosphonium hexafluorophosphate, PyAOP)克服空间位 阻, 使甲胺化可以对 $\alpha-2,3$-和 $\alpha-2,6$-连接的唾液酸在温 和条件下进行完全衍生 ${ }^{[65]}$.

但是以上所述全甲基化等衍生方法无法实现对 N糖链唾液酸连接异构体的识别和检测, 所以近年来, 研 究者们发展了更多基于质谱的针对 $\mathrm{N}$-糖链唾液酸连接 异构体进行识别检测的衍生方法.

\subsubsection{N-糖链唾液酸连接异构体的一步识别衍生分析 方法}

衍生法在区分 $\mathrm{N}$-糖链唾液酸连接异构体方面应用 很广泛. Wheeler 等[66]通过缩合剂 4-(4,6-二甲氧基三嗪2- 基 )-4- 甲基吗啉盐酸盐 (4-(4,6-dimethoxy-1,3,5triazin-2-yl)-4-methyl morpholinium chloriden-hydrate, DMT-MM), 使唾液酸完成连接特异性衍生, $\alpha-2,6$-连接 的唾液酸被甲酯化, 分子量增加 $14 \mathrm{Da}, \alpha$-2,3-连接的唾 液酸化糖链生成内酯, 分子量减少 $18 \mathrm{Da}$, 两者相差 32 $\mathrm{Da}$, 在质谱中可以进行区分. Reiding 等 ${ }^{[67]}$ 通过在乙醇溶 剂中使用 1-(3-二甲基氨基丙基)-3-乙基碳化二亚胺 (1-(3-(dimethylamino)propyl)-3-ethylcarbodiimide, EDC) 实现唾液酸异构体的识别性衍生, $\alpha-2,3$-连接的唾液酸 形成内酯, $\alpha-2,6$-连接的唾液酸生成乙酯. 但这两种方式 
都是将 $\alpha-2,3$-连接的唾液酸转化为在水中不太稳定的内 酯, 对唾液酸异构体的相对定量造成了困难, 很多研究 者对其进行了改进.

Jin 等 ${ }^{[68]}$ 用催化剂 1-羟基苯并三氮唑一水合物 (1-hydroxybenzotriazole monohydrate, HOBt)进行衍生, 他们用非氝代 $\left(d_{0}\right)$ 或気代 $\left(d_{5}\right)$ 的苯胺先进行了非识别性 衍生, 根据谱图中成对存在的峰, 确定唾液酸残基的个 数. 之后他们用 $\mathrm{A}$ 溶液(溶有 $250 \mathrm{mmol} / \mathrm{L} \mathrm{EDC}$ 和 500 $\mathrm{mmol} / \mathrm{L} \mathrm{HOBt}$ 的 DMSO 溶液)和 B 溶液(溶有 $500 \mathrm{mmol} / \mathrm{L}$ $\mathrm{EDC}, 1 \mathrm{~mol} / \mathrm{L} \mathrm{HOBt}$ 和 $500 \mathrm{mmol} / \mathrm{L}$ 気代苯胺的 $\mathrm{DMSO}$ 溶液)进行唾液酸异构体的识别性衍生, 其中, $\alpha-2,3$-连 接的唾液酸形成内酯, 质量减少 $18 \mathrm{Da}, \alpha-2,6$-连接的唾 液酸被氝代的苯胺酰胺化, 质量增加 $80 \mathrm{Da}$, 两者的质 量差为 $98 \mathrm{Da}$, 通过反相高效液相色谱(reversed-phase high performance liquid chromatography coupling, RP-HPLC)和质谱联用, 可以在谱图中区分 $\mathrm{N}$-糖链的唾 液酸连接异构体, 他们用该方法鉴定了牛 $\operatorname{IgG}$ 中 12 个 $\alpha-2,6$-连接的唾液酸化 $\mathrm{N}$-糖链和兔 $\operatorname{IgG}$ 中 30 个 $\alpha-2,6-$ 连接的唾液酸化 $\mathrm{N}$-糖链.

Pongracz 等 ${ }^{[69]}$ 以 $\mathrm{HOBt}$ 为催化剂, 将 $\mathrm{N}$-糖链的两种 唾液酸连接异构体在乙醇溶液中进行反应, $\alpha-2,3$-连接 的唾液酸内酯化反应产物使分子量减少 $18 \mathrm{Da}, \alpha-2,6$-连 接的唾液酸乙酯化反应产物使分子量增加 $28 \mathrm{Da}$, 两者 质量差为 $46 \mathrm{Da}$, 他们考察了 6 种催化剂(6-氯-1-羟基苯 并三唑 (6-chloro-1-hydroxybenzotriazole, 6-Cl-HOBt), 1-羟基-6-(三氟甲基)苯并三唑(1-hydroxy-6-(trifluoromethyl)benzotriazole, $6-\mathrm{CF}_{3}-\mathrm{HOBt}$ ), 羟基三唑并 [4,5-b] 吡啶(hydroxytriazolo[4,5-b]pyridine, HOAt), 1-着基三唑4-羧酸乙酯(ethyl 1-hydroxytriazole-4-carboxylate, HOCt) 和 (2E)-2-氭基-2-羟基亚氨基乙酸乙酯 (ethyl (2E)-2cyano-2-hydroxyiminoacetate, Oxyma Pure)的催化性能. 结果显示: Oxyma pure 催化剂毒性较高; HOCt 的反应效
率低; HOAt 在 $\alpha-2,6$-连接的唾液酸糖链异构体的反应中 特异性较低, 而 HOBt、6-Cl-HOBt 和 $6-\mathrm{CF}_{3}-\mathrm{HOBt}$ 的衍 生化效率最高, 并有比其他催化剂更好的反应特异性, 所以，6-Cl-HOBt 和 $6-\mathrm{CF}_{3}-\mathrm{HOBt}$ 可用于替代 HOBt 与 EDC 和乙醇进行唾液酸连接异构体识别性衍生化的步 骤，因替代催化剂的物理化学性质有所不同，反应溶剂 可选择的种类也变得更为多样.

\subsubsection{N-糖链唾液酸连接异构体的两步识别衍生分析 方法}

两步识别衍生法是在一步识别衍生法的基础上发 展的. 在第一步衍生的基础上, 引入第二步反应使 $\alpha-2,3$-连接的唾液酸形成稳定的酰胺等结构. Alley 等 ${ }^{[59]}$ 开发了唾液酸特异性内酯化/酰胺化反应, 第一步用 DMT-MM 处理糖链唾液酸异构体, $\alpha-2,3$-连接的唾液酸 的羧酸形成环状内酯, $\alpha-2,6$-连接的唾液酸结构形成酰 胺, 第二步用碘甲烷将两种唾液酸结构都进行全甲基 化, 最后的质量差为 $13 \mathrm{Da}$ (图 2), 他们检测了健康志愿 者和乳腺癌晚期患者各 10 例血清样本, 发现在乳腺癌 患者的血清样本中 $\alpha-2,6$-连接的唾液酸化糖链异构体含 量较高, 而健康志愿者血清样本中的 $\alpha-2,3$-连接的唾液 酸化糖链异构体含量较高.

$\mathrm{Li}$ 等 ${ }^{[70]}$ 开发了固相两步衍生化(solid-phase two step derivatization, STSD)方法, 先将 $\alpha-2,6$-连接的唾液酸转 化为乙酯, 质量增加 $28 \mathrm{Da}$, 同时 $\alpha-2,3$-连接的唾液酸形 成内酯; 然后内酯在弱碱性条件下完全水解, $\alpha-2,6$-连接 的唾液酸生成的乙酯不变; 在 PyAOP 存在的情况下, $\alpha$-2,3-连接的唾液酸与甲胺盐反应形成甲酰胺, 质量增 量为 $13 \mathrm{Da}$, 两者质量差为 $15 \mathrm{Da}$, 该方法反应条件比较 温和，他们用该方法分析发现，在人血清 $\operatorname{IgG}$ 中，主要 是 $\alpha-2,6$-连接的唾液酸化 $\mathrm{N}$-糖链, 而在人造单克隆抗体 的 $\operatorname{IgG}$ 中, 主要是 $\alpha-2,3$-连接的唾液酸化 $\mathrm{N}$-糖链.

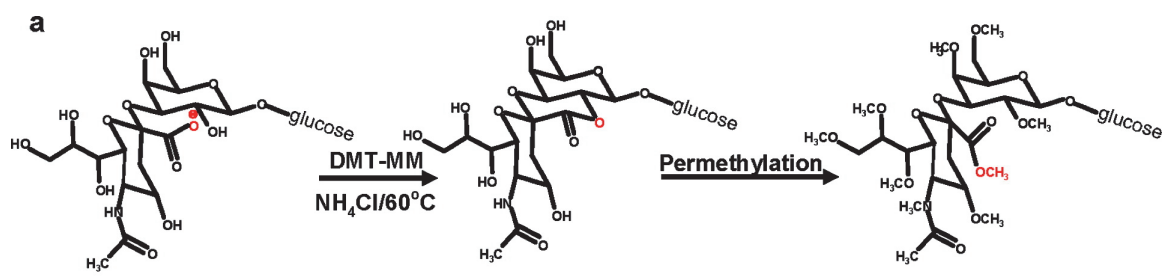

b

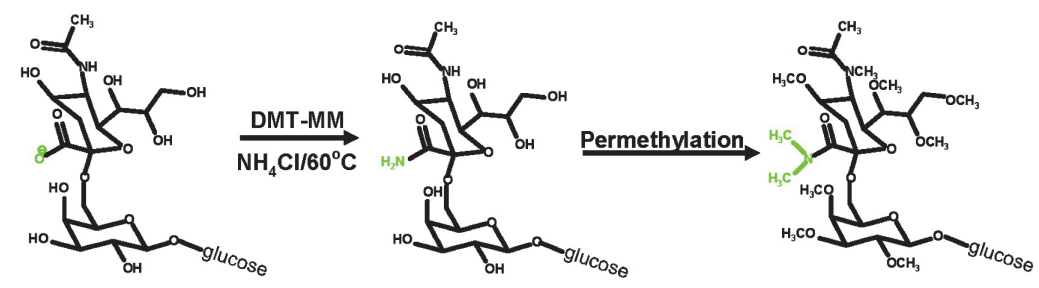

图 2 特异性内酯化/酰胺化反应 ${ }^{[59]}$

Figure 2 Lactonization/amidation reaction for derivatizing glycans ${ }^{[99]}$ 
Holst 等[71]研究了一种识别性衍生方法, 第一步只 有 EDC、HOBt、DMSO 和二甲胺存在, $\alpha-2,6$-连接的唾 液酸形成二甲基酰胺, 质量增加 $27 \mathrm{Da}, \alpha$-2,3-连接的唾 液酸与相邻的半乳糖反应生成内酯; 第二步加入氢氧化 铵, 内酯水解, 羧酸形成酰胺, 质量减少 $1 \mathrm{Da}$, 异构体 之间的质量差为 $28 \mathrm{Da}$ (图 3). 他们用标准唾液酸化 N糖链验证了这一方法, 该方法对 $\alpha$-2,3-和 $\alpha$-2,6-连接唾 液酸的标记选择性分别为 $98.3 \%$ 和 $99.2 \%$. 他们利用原 位衍生的方法对福尔马林固定石蜡包埋的结直肠癌 (colorectal cancer, CRC) 患者的平滑肌肉瘤 (leiomyosarcoma) 组织进行处理, 并进行 MALDI 质谱成 像后他们发现, 含有 $\alpha-2,3$-连接唾液酸的 $\mathrm{N}$-糖链主要存 在于肿瘤区域, 而含有 $\alpha-2,6$-连接唾液酸的 $N$-糖链主要 存在于坏死组织、富含胶原区等. Zhou 等[72]用胎球蛋白 和重组人乳铁蛋白验证了相同的方法, 该方法对复杂的 蛋白质样本效果良好, 并有可靠的准确性和稳定性, 并 可用于高通量的 N-糖链唾液酸连接异构体研究.

Nishikaze 等[73]开发了唾液酸连接特异性烷基酰胺 化反应(sialic acid linkage specific alkylamidation, SAL$\mathrm{SA})$, 第一步烷基酰胺化使用 $\mathrm{EDC} \cdot \mathrm{HCl} 、 \mathrm{HOBt}$ 、盐酸异 丙胺(isopropylamine hydrochloride, iPA- $\mathrm{HCl}$ )、DMSO 等 进行衍生反应, $\alpha-2,6$-连接的唾液酸形成异丙酰胺, 质量
增加 $41 \mathrm{Da}, \alpha-2,3$-连接的唾液酸与相邻的半乳糖形成内 酯，质量减少 $18 \mathrm{Da}$; 第二步烷基酰胺化使用 $1 \mathrm{H}$-苯并三 唑-1- 基氧三吡咯烷基六氟磷酸盐 (1H-benzotriazol1-yloxytripyrroli-dinophosphonium hexafluorophosphate, PyBOP)、甲胺盐酸盐( $\mathrm{MA}-\mathrm{HCl}) 、 N$-甲基吗啉 $(N$-methylmorpholine, N-MM)和 DMSO 进行反应, $\alpha$-2,6-连接的唾 液酸不变, $\alpha-2,3$-连接的唾液酸形成的内酯水解并进行 甲胺化, 质量增加 $13 \mathrm{Da}$, 最终两者质量差为 $28 \mathrm{Da}$ (图 4). SALSA 的衍生特异性较好, 他们用含两个唾液酸的 标准 $\mathrm{N}$-糖链验证了该方法, 其对 $\alpha$-2,3-和 $\alpha$-2,6-连接唾 液酸的反应选择性分别为 $95.1 \%$ 和 $99.5 \%$. 他们用该方 法检测比较发现人血清 $\mathrm{IgG}$ 主要含有 $\alpha-2,6$-连接的唾液 酸化 $\mathrm{N}$-糖链, 抗体药物利妥昔单抗(rituximab)仅含有 $\alpha$-2,3-连接的唾液酸化 N-糖链. 但在 SALSA 法中, 样品 里潜在的污染物可能会降低衍生化效率或引起副反应. 特别是应避免氨的污染, 在缩合试剂存在的情况下, 氨 易与羧基反应，不利于酰胺的形成，由此反应过程中需 要 HILIC 纯化, 但纯化过程既费时又会造成样品丢失, 所以之后该团队结合 SALSA 方法和酰肼微珠(hydrazide bead)开发 “一锅法” 唾液酸识别衍生方法, 省去 SALSA 法中预脱盐和连续的 HILIC 纯化步骤, 并能够从复杂的 生物样品中直接纯化糖链.
A

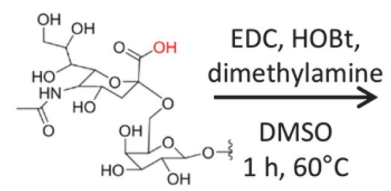

$\alpha 2,6$-linked sialic acid

B

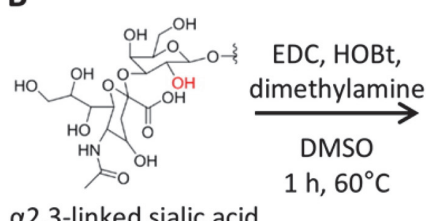

$\alpha 2,3$-linked sialic acid
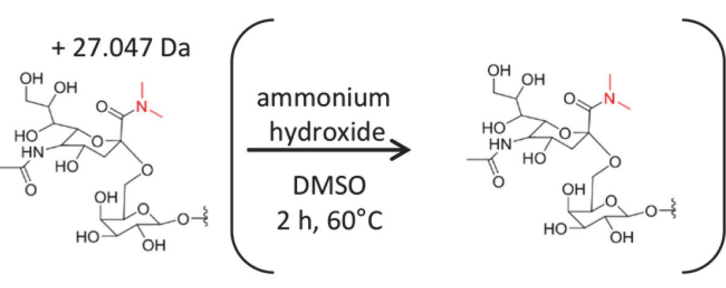

$-0.984 \mathrm{Da}$
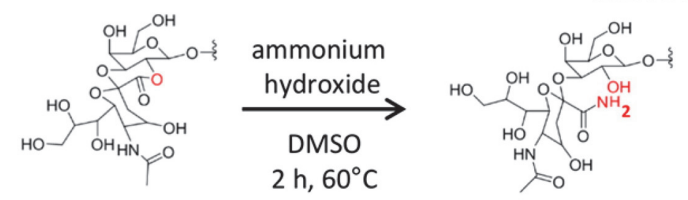

图 3 唾液酸化 $\mathrm{N}$-糖链的特异性二甲胺/氢氧化铵反应 ${ }^{[71]}$

Figure 3 Dimethylamine/ammonium hydroxide reaction of sialylated N-glycans ${ }^{[71]}$

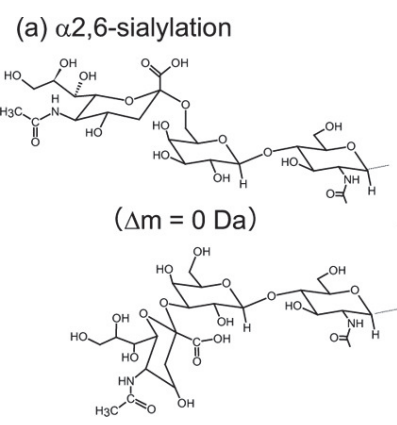

(b) $\alpha 2,3$-sialylation
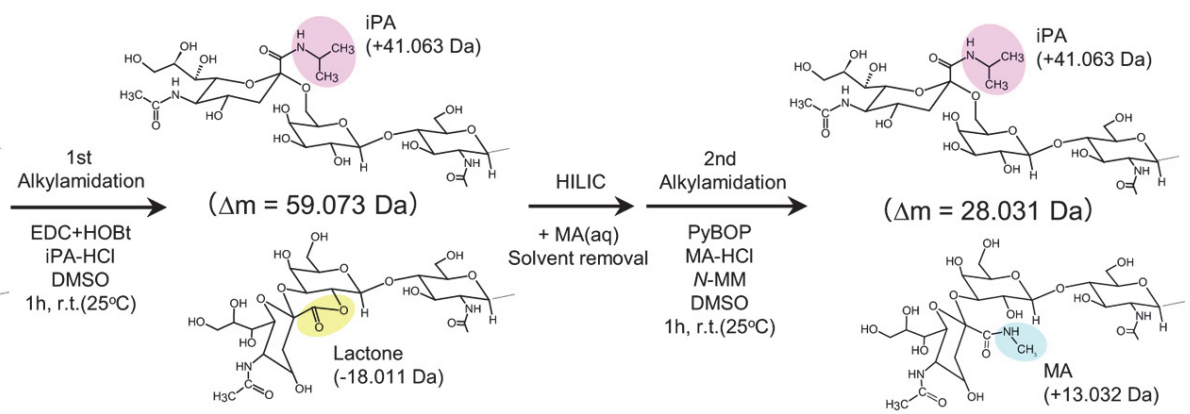

图 4 唾液酸化 N-糖链的特异性烷基酰胺化反应 ${ }^{[73]}$

Figure 4 Alkylamidation of sialylated N-glycans ${ }^{[73]}$ 
Suzuki 等 ${ }^{[74]}$ 也改进了 SALSA 方法, 以减少副产物 的产生, 他们将 $\alpha 1-\mathrm{AGP}$ 酶解得到的混合 N-糖链用 2氨基吡啶(2-aminopyridine, PA)标记, 用 LC-MS-MS/MS 对样品进行分析, 同时用苂光检测器进行检测, PA-N糖链的反应效率均 $>94 \%$, 副产物很少, 并可根据各峰 的积分苂光强度估算 $\alpha-2,3$-和 $\alpha-2,6$-连接的唾液酸异构 体的相对含量. 他们在第二步衍生中用甲胺溶液代替了 甲胺盐酸盐后获得的反应效率较高( $94.1 \%$ ～98.4\%), 误 转化率低( $1.4 \% \sim 3.9 \%)$, 因此误判糖链的风险较低, 所 以适用于未知样品. 同时他们还改进了双酰胺化方法, 用甲胺水溶液进行第二步烷基酰胺化，由于省去了第一 次烷基酰胺化后的 HILIC 纯化, 变得方便省时, 并降低 了样品的丢失, 反应效率(93.3\% 94.5\%)较高, 但对于 低浓度的糖链衍生的副产物还比较明显(3.2\% 4.7\%).

Furukawa 等 ${ }^{[75]}$ 开发了内酯驱动的识别唾液酸连接 异构体的酯 - 酰胺衍生化方法 (lactone-driven ester-to-amide derivatization for sialic acid linkage-specific alkylamidation, LEAD-SALSA), 他们将糖链上的 $\alpha-2,3-$ 和 $\alpha-2,6$-连接的唾液酸残基先进行甲酯化, 再用乙胺通 过内酯开环氨解反应使 $\alpha-2,3$-连接的唾液酸甲酯形成酰 胺, 而 $\alpha-2,6$-连接的唾液酸残基保持甲酯不变(图 5). 他 们分析了 6、12、21 和 24 个月大的小鼠心肌组织中二 唾液酸二分支糖链 $(\mathrm{A} 2 \mathrm{~F})$ 的相对含量, 在进行甲酯化和 用丙胺进行酯-酰胺化并用 HILIC 进行纯化后, $\alpha-2,3$-连
接的唾液酸比 $\alpha$-2,6-连接的唾液酸质量增大 $27 \mathrm{Da}$, 发 现在 21 个月大的小鼠中, $\alpha-2,6$-连接唾液酸糖链的相对 含量显著增加.

以上识别性衍生方法都将两种类型的唾液酸转化 为不同化学性质的化合物, 这会导致质谱分析中异构体 的离子化效率不同，所以信号强度的比率会偏离实际比 率, 这会使定量出现一定的误差 ${ }^{[76]}$. 我们 ${ }^{[76]}$ 利用稳定同 位素标记的甲胺盐酸盐 $\left(\mathrm{CH}_{3} \mathrm{NH}_{2} \bullet \mathrm{HCl}\right.$ 或 $d_{0}-\mathrm{MA} \bullet \mathrm{HCl}$ 和 $\mathrm{CD}_{3} \mathrm{NH}_{2} \cdot \mathrm{HCl}$ 或 $d_{3}-\mathrm{MA} \cdot \mathrm{HCl}$ ) 对糖链进行顺序衍生化, 对 于正向标记，先使用 $d_{0}-\mathrm{MA} \cdot \mathrm{HCl} 、 \mathrm{EDC} \cdot \mathrm{HCl}$ 和 $\mathrm{HOBt}$ 使 $\alpha$-2,6-连接的唾液酸甲胺酰胺化, $\alpha$-2,3-连接的唾液酸形 成内酯, 再使用 $d_{3}-\mathrm{MA} \cdot \mathrm{HCl}$ 和 PyAOP 使 $\alpha-2,3$-连接的唾 液酸甲胺酰胺化, 从而得到轻标记的 $\alpha-2,6$-连接的唾液 酸和重标记的 $\alpha-2,3$-连接的唾液酸; 反向标记先用 $d_{3}-\mathrm{MA} \cdot \mathrm{HCl}$ 后用 $d_{0}-\mathrm{MA} \cdot \mathrm{HCl}$, 生成轻标记的 $\alpha-2,3$-连接 的唾液酸和重标记的 $\alpha-2,6-$ 连接的唾液酸, 每个连接方 式不同的唾液酸会造成 $3 \mathrm{Da}$ 的质量差(图 6), 由于两步 标记的试剂仅是稳定同位素的差异，因此在质谱分析中 两种唾液酸链接异构体的离子化效率相同. 我们先用含 有一个唾液酸的标准 N-糖链验证这一方法, 其对 $\alpha-2,3-$ 和 $\alpha-2,6$-连接的唾液酸衍生化选择性为 $98.81 \%$ 与 $99.81 \%$, 对于含两个唾液酸的 $\mathrm{N}$-糖链, 其对 $\alpha-2,3$-和 $\alpha$-2,6-连接的唾液酸衍生化选择性为 $98.11 \%$ 与 $98.86 \%$. 我们通过 MALDI-MS 检测 CRC 患者血清的 $\mathrm{N}$-糖链, 检
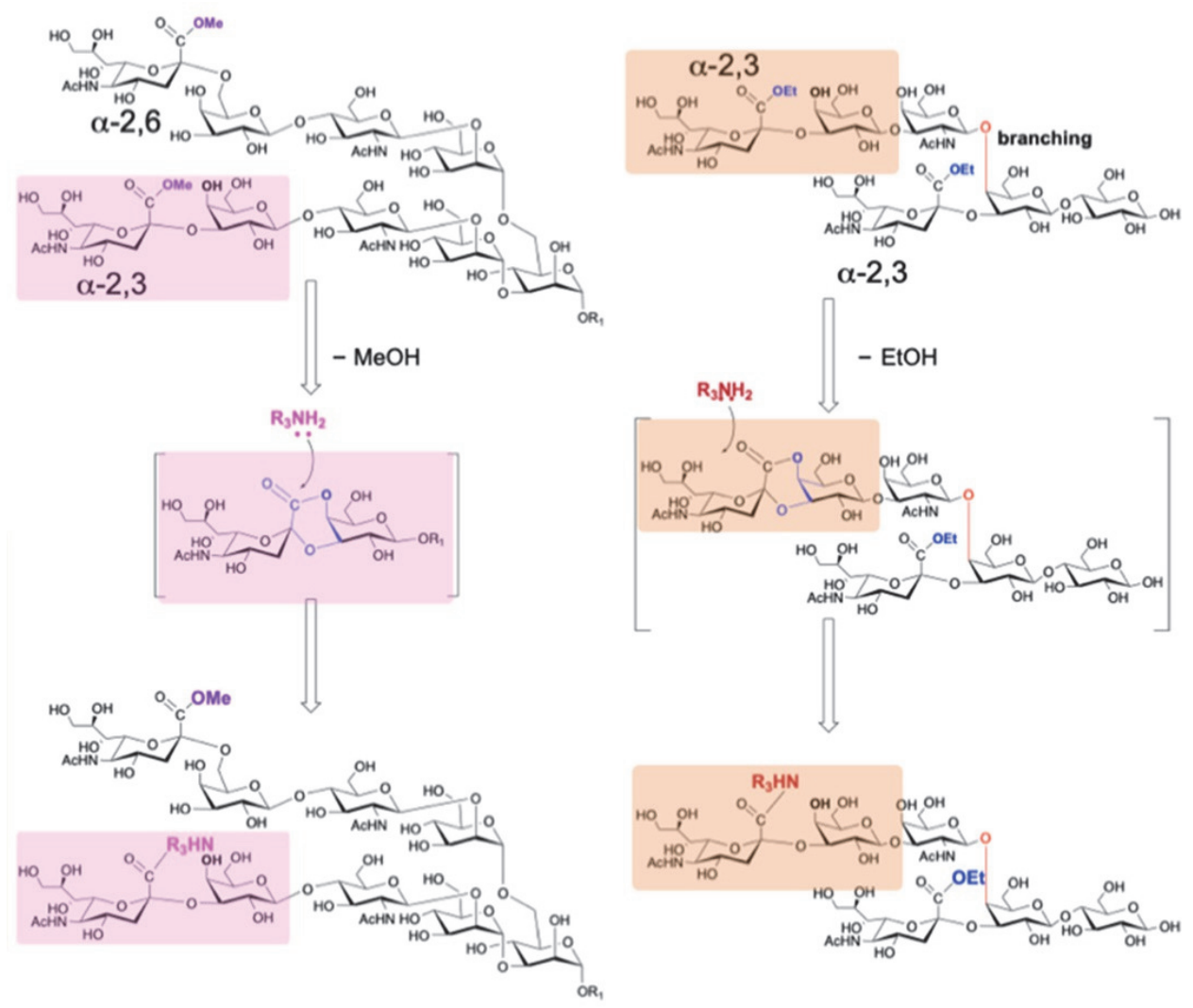

图 5 内酯驱动的酯-酰胺衍生化反应 ${ }^{[75]}$

Figure 5 Lactone-driven ester-amide derivatization reaction ${ }^{[75]}$ 


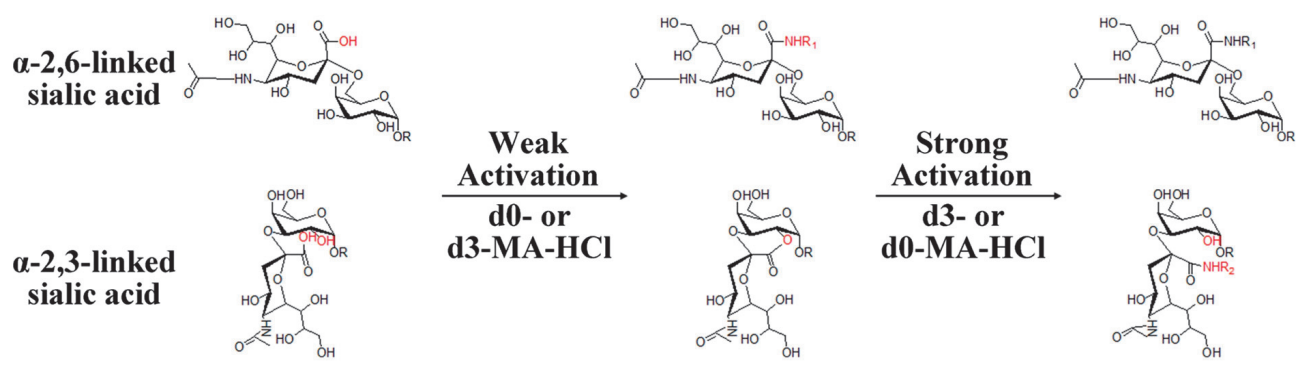

For forward labelling (d0-MA-HCl followed by d3-MA-HCl), R1 $=\mathrm{CH}_{3}, \mathbf{R 2}=\mathbf{C D}$ For reverse labelling (d3-MA-HCl followed by d0-MA-HCl), R1 $=\mathrm{CD}_{3}, \mathrm{R} 2=\mathrm{CH}_{3}$

图 6 唾液酸化糖链的两步甲基酰胺化 ${ }^{[76]}$

Figure 6 Two-step methylamidation of sialylated glycans ${ }^{[76]}$

测到 62 种 $\mathrm{N}$-糖链, 包括 34 个唾液酸化 $\mathrm{N}$-糖链和 8 对 连接异构体, 其中 6 对唾液酸异构体的 $\alpha-2,6$-唾液酸水 平发生了显著上升, 并且 CRC 患者血清 $\mathrm{N}$-糖链中总 $\alpha-2,6$-唾液酸水平增加, 所以, 特定的连接异构体可能 是诊断 CRC 的潜在标志物.

\section{4 总结与展望}

糖链的唾液酸连接异构体在生理和病理过程中的 作用不同，进行唾液酸异构体的检测和定量分析在探究 其生理功能，进行相关疾病诊断、制定生物制药指标等 方面有重要的意义. 近年来, $\mathrm{N}$-糖链的唾液酸连接异构 体的检测有了较大的发展. 非衍生方法方面开发了基于 高效阴离子交换色谱, 基于多孔石墨化碳柱, 基于毛细 管电泳的方法和基于离子淌度质谱的方法, 其中基于多 孔石墨化碳柱的非衍生法是应用最为广泛的方法.

近年来, N-糖链唾液酸连接异构体的识别性衍生方 法得到了较大的发展, 特别是基于质谱的检测手段使高 灵敏度、高选择性检测得以实现. 目前的识别性衍生方 法的主要依据是 $\alpha-2,3$-连接和 $\alpha-2,6$-连接唾液酸的不同 反应活性, 而且研究者们考虑了 $\alpha-2,3$-连接唾液酸的稳 定酰胺化, 从一步衍生发展为两步衍生, 我们组还考虑 到, 衍生后形成的物质不同会使其在质谱分析的离子化 效率不同, 继而发展了稳定同位素的衍生方法.

用来衍生唾液酸的亲核试剂已成为识别性反应的 关键因素之一, 未来将会发展更多衍生相关的亲核试 剂, 反应过程也会更加优化, 使衍生效率进一步提高, 副产物进一步减少. 同时识别性衍生方法在未来将会结 合更多相关方法共同提高检测的灵敏度和选择性, 并会 在原位衍生和组织成像方面得到更为长足的发展. N-糖 链唾液酸连接异构体是经过酶催化形成的, 但其机理仍 需要进一步探究, 这需要检测唾液酸连接异构体的深度 覆盖以及开展唾液酸糖基转移酶与糖链变化之间的关 联性的研究. 此外, 基于 $\mathrm{N}-$ 糖链唾液酸连接异构体的鉴 别检测在癌症检测中的潜力已经开始显现, 在后续工作 中, 发展适用于临床检测的、快速高效、高通量的检测 方法也是一个较为重要的研究方向. 更进一步地, 由于
$\mathrm{N}$-糖链唾液酸连接异构体在细胞表面分布的不同，未来 进行单细胞唾液酸连接异构体分析可能会进一步提示 细胞异质性与糖链的关系.

\section{作者简介}

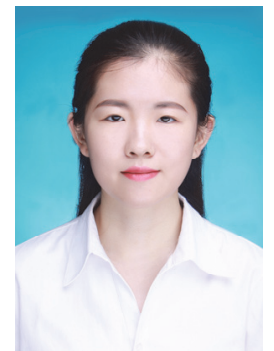

李月悦，女，汉族，2020 年本科毕业于华东理工大学，随 后进入复旦大学生物医学研究院陆豪杰课题组攻读博士学位, 主要从事于唾液酸化 N-糖链连接异构体的分离与定量方法研 究.

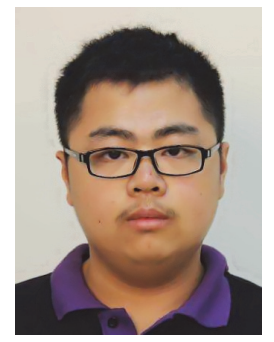

彭叶, 男, 汉族. 2016 年本科毕业于复旦大学化学系, 随 后进入复旦大学生物医学研究院陆豪杰课题组攻读博士学位. 主要参与基于质谱的蛋白质糖基化分析方法学研究.

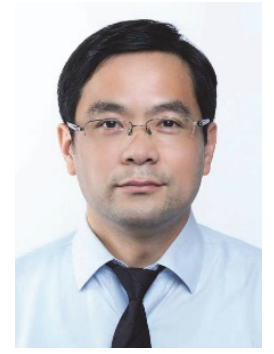

陆豪杰, 男, 汉族, 复旦大学教授, 博士生导师, 于 $1992 \sim 1996$ 年在厦门大学化学系就读, 获得学士学位; 于 
1996 2001 年在中国科学院兰州化学物理研究所硕博连读, 获得博士学位; 于 2001 2003 年在中国科学院上海有机化学 研究所从事博士后研究; 自 2003 年至今, 在复旦大学化学系 和复旦大学生物医学研究院从事生物质谱新技术和新方法的 研究.

\section{References}

[1] Apweiler, R.; Hermjakob, H.; Sharon, N. Biochim. Biophys. Acta 1999, 1473, 4.

[2] Dutta, D.; Mandal, C.; Mandal, C. Biochim. Biophys. Acta 2017, $1861,3096$.

[3] Moremen, K. W.; Tiemeyer, M.; Nairn, A. V. Nat. Rev. Mol. Cell Biol. 2012, 13, 448.

[4] Xiong, Y.-Y.; Chen, Y.-L.; Jv, H.-X. Acta Chim. Sinica 2019, 77, 1221 (in Chinese). (熊莹莹, 陈云龙, 鞠熀先, 化学学报, 2019, 77, 1221.)

[5] Wang, X.-D.; Liu, Y.-J.; Li, F.-J.; Li, Z.-L. Chin. Chem. Lett. 2017, $28,1018$.

[6] Demetriou, M.; Granovsky, M.; Quaggin, S.; Dennis, J. W. Nature 2001, 409, 733.

[7] Ladenson, R. P.; Schwartz, S. O.; Ivy, A. C. Am. J. Med. Sci. 1949, $217,194$.

[8] Zhou, S.; Hu, Y.; Veillon, L.; Snovida, S. I.; Rogers, J. C.; Saba, J.; Mechref, Y. Anal. Chem. 2016, 88, 7515 .

[9] Zhou, X.-M.; Yang, S.; Yang, G.-L.; Tan, Z.-Q.; Guan, F. Chin. Chem. Lett. 2019, 30, 676.

[10] Chandrasekaran, A.; Srinivasan, A.; Raman, R.; Viswanathan, K.; Raguram, S.; Tumpey, T. M.; Sasisekharan, V.; Sasisekharan, R. Nat. Biotechnol. 2008, 26, 107.

[11] Zlatina, K.; Saftenberger, M.; Kuhnle, A.; Galuska, C. E.; Gartner, U.; Rebl, A.; Oster, M.; Vernunft, A.; Galuska, S. P. Int. J. Mol. Sci. 2018, 19,10 .

[12] Lv, J.; Wang, Z. X.; Li, F.; Zhang, Y.; Lu, H. J. Chem. Commun. 2019, 55, 14339.

[13] Abercrombie, M.; Ambrose, E. J. Cancer Res. 1962, 22, 525.

[14] Forrester, J. A.; Ambrose, E. J.; Stoker, M. G. P. Nature 1964, 201, 945.

[15] Cazet, A.; Julien, S.; Bobowski, M.; Burchell, J.; Delannoy, P. Breast Cancer Res. 2010, 12, 204.

[16] Schultz, M. J.; Swindall, A. F.; Bellis, S. L. Cancer Metastasis Rev. 2012, 31, 501.

[17] Bray, F.; Ferlay, J.; Soerjomataram, I.; Siegel, R. L.; Torre, L. A.; Jemal, A. CA Cancer J. Clin. 2018, 68, 394.

[18] Leone, J. P.; Leone, B. A. Exp. Hematol. Oncol. 2015, 4, 10.

[19] Peng, W.; Goli, M.; Mirzaei, P.; Mechref, Y. J. Proteome Res. 2019, 18,3731

[20] Kim, Y. J.; Varki, A. Glycoconjugate J. 1997, 14, 569.

[21] Sato, C.; Kitajima, K. J. Biochem. 2013, 154, 115.

[22] Galuska, C. E.; Lutteke, T.; Galuska, S. P. Biology 2017, 6, 27.

[23] Lan, Y.; Hao, C.; Zeng, X.; He, Y. L.; Zeng, P. J.; Guo, Z. H.; Zhang, L. J. Am. J. Cancer Res. 2016, 6, 2390.

[24] Ferrer-Batalle, M.; Llop, E.; Ramirez, M.; Aleixandre, R. N.; Saez, M.; Comet, J.; de Llorens, R.; Peracaula, R. Int. J. Mol. Sci. 2017, 18,845 .

[25] Vasseur, J. A.; Goetz, J. A.; Alley, W. R., Jr.; Novotny, M. V. Glycobiology 2012, 22, 1684.

[26] Siegel, R. L.; Miller, K. D.; Jemal, A. CA-Cancer J. Clin. 2017, 67, 7.

[27] Mathieu, K. B.; Bedi, D. G.; Thrower, S. L.; Qayyum, A.; Bast, R. C. Ultrasound Obstet. Gynecol. 2018, 51, 293.

[28] Dedova, T.; Braicu, E. I.; Sehouli, J.; Blanchard, V. Front. Oncol. 2019, 9,261

[29] Llop, E.; Ferrer-Batalle, M.; Barrabes, S.; Guerrero, P. E.; Ramirez, M.; Saldova, R.; Rudd, P. M.; Aleixandre, R. N.; Comet, J.; de Llorens, R.; Peracaula, R. Theranostics 2018, 8, 746.

[30] Tousi, F.; Bones, J.; Hancock, W. S.; Hincapie, M. Anal. Chem. 2013, $85,8421$.

[31] Wang, P. H.; Lee, W. L.; Lee, Y. R.; Juang, C. M.; Chen, Y. J.; Chao, H. T.; Tsai, Y. C.; Yuan, C. C. Gynecol. Oncol. 2003, 89, 395.

[32] Swindall, A. F.; Londono-Joshi, A. I.; Schultz, M. J.; Fineberg, N.; Buchsbaum, D. J.; Bellis, S. L. Cancer Res. 2013, 73, 2368.

[33] Yamamoto, H.; Oviedo, A.; Sweeley, C.; Saito, T.; Moskal, J. R.
Cancer Res. 2001, 61, 6822.

[34] Julien, S.; Adriaenssens, E.; Ottenberg, K.; Furlan, A.; Courtand, G.; Vercoutter-Edouart, A. S.; Hanisch, F. G.; Delannoy, P.; Le Bourhis, X. Glycobiology 2006, 16, 54 .

[35] Sewell, R.; Backstrom, M.; Dalziel, M.; Gschmeissner, S.; Karlsson, H.; Noll, T.; Gatgens, J.; Clausen, H.; Hansson, G. C.; Burchell, J.; Taylor-Papadimitriou, J. J. Biol. Chem. 2006, 281, 3586.

[36] Harvey, D. J. Mass Spectrom. Rev. 1999, 18, 349.

[37] Harvey, D. J. Proteomics 2001, 1, 311 .

[38] Ruhaak, L. R.; Xu, G. G.; Li, Q. Y.; Goonatilleke, E.; Lebrilla, C. B. Chem. Rev. 2018, 118, 7886.

[39] Cataldi, T. R. I.; Campa, C.; De Benedetto, G. E. Fresenius J. Anal. Chem. 2000, 368, 739.

[40] Behan, J. L.; Smith, K. D. Biomed. Chromatogr. 2011, 25, 39.

[41] Zamze, S.; Harvey, D. J.; Chen, Y. J.; Guile, G. R.; Dwek, R. A.; Wing, D. R. Eur. J. Biochem. 1998, 258, 243.

[42] Bones, J.; McLoughlin, N.; Hilliard, M.; Wynne, K.; Karger, B. L.; Rudd, P. M. Anal. Chem. 2011, 83, 4154.

[43] Townsend, R. R.; Hardy, M. R.; Cumming, D. A.; Carver, J. P.; Bendiak, B. Anal. Biochem. 1989, 182, 1.

[44] Maier, M.; Reusch, D.; Bruggink, C.; Bulau, P.; Wuhrer, M.; Molhoj, M. J. Chromatogr. B Analyt. Technol. Biomed. Life Sci. 2016 1033-1034, 342.

[45] Ruhaak, L. R.; Deelder, A. M.; Wuhrer, M. Anal. Bioanal. Chem. 2009, 394, 163.

[46] Ruhaak, L. R.; Zauner, G.; Huhn, C.; Bruggink, C.; Deelder, A. M.; Wuhrer, M. Anal. Bioanal. Chem. 2010, 397, 3457.

[47] West, C.; Elfakir, C.; Lafosse, M. J. Chromatogr. A 2010, 1217, 3201.

[48] Stavenhagen, K.; Kolarich, D.; Wuhrer, M. Chromatographia 2015, $78,307$.

[49] Pabst, M.; Bondili, J. S.; Stadlmann, J.; Mach, L.; Altmann, F. Anal. Chem. 2007, 79, 5051.

[50] Ashwood, C.; Lin, C. H.; Thaysen-Andersen, M.; Packer, N. H. $J$ Am. Soc. Mass Spectrom. 2018, 29, 1194.

[51] Anugraham, M.; Jacob, F.; Nixdorf, S.; Everest-Dass, A. V.; Heinzelmann-Schwarz, V.; Packer, N. H. Mol. Cell Proteomics 2014, $13,2213$.

[52] Kammeijer, G. S. M.; Jansen, B. C.; Kohler, I.; Heemskerk, A. A. M.; Mayboroda, O. A.; Hensbergen, P. J.; Schappler, J.; Wuhrer, M. Sci. Rep. 2017, 7, 3733.

[53] Fuguet, E.; Ràfols, C.; Bosch, E.; Rosés, M. Chem. Biodivers. 2009, $6,1822$.

[54] May, J. C.; McLean, J. A. Anal. Chem. 2015, 87, 1422.

[55] Manz, C.; Pagel, K. Curr. Opin. Chem. Biol. 2018, 42, 16.

[56] Hinneburg, H.; Hofmann, J.; Struwe, W. B.; Thader, A.; Altmann, F.; Varon Silva, D.; Seeberger, P. H.; Pagel, K.; Kolarich, D. Chem. Commun. 2016, 52, 4381.

[57] Barroso, A.; Gimenez, E.; Konijnenberg, A.; Sancho, J.; Sanz-Nebot, V.; Sobott, F. J. Proteomics 2018, 173, 22.

[58] Lane, C. S.; McManus, K.; Widdowson, P.; Flowers, S. A.; Powell, G.; Anderson, I.; Campbell, J. L. Anal. Chem. 2019, 91, 9916.

[59] Alley, W. R., Jr.; Novotny, M. V. J. Proteome Res. 2010, 9, 3062.

[60] Ciucanu, I.; Costello, C. E. J. Am. Chem. Soc. 2003, 125, 16213.

[61] Powell, A. K.; Harvey, D. J. Rapid Commun. Mass Spectrom. 1996, $10,1027$.

[62] Sekiya, S.; Wada, Y.; Tanaka, K. Anal. Chem. 2005, 77, 4962.

[63] Toyoda, M.; Ito, H.; Matsuno, Y. K.; Narimatsu, H.; Kameyama, A. Anal. Chem. 2008, 80, 5211.

[64] Shah, P.; Yang, S.; Sun, S. S.; Aiyetan, P.; Yarema, K. J.; Zhang, H. Anal. Chem. 2013, 85, 3606.

[65] Liu, X.; Qiu, H. Y.; Lee, R. K.; Chen, W. X.; Li, J. J. Anal. Chem. 2010, $82,8300$.

[66] Wheeler, S. F.; Domann, P.; Harvey, D. J. Rapid Commun. Mass Spectrom. 2009, 23, 303.

[67] Reiding, K. R.; Blank, D.; Kuijper, D. M.; Deelder, A. M.; Wuhrer, M. Anal. Chem. 2014, 86, 5784 .

[68] Jin, W.; Wang, C.; Yang, M.; Wei, M.; Huang, L.; Wang, Z. Anal. Chem. 2019, 91, 10492

[69] Pongracz, T.; Wuhrer, M.; de Haan, N. Molecules 2019, 24, 3617.

[70] Li, H.; Gao, W.; Feng, X.; Liu, B. F.; Liu, X. Anal. Chim. Acta 2016, 924, 77.

[71] Holst, S.; Heijs, B.; de Haan, N.; van Zeijl, R. J.; Briaire-de Bruijn, I. H.; van Pelt, G. W.; Mehta, A. S.; Angel, P. M.; Mesker, W. E.; Tollenaar, R. A.; Drake, R. R.; Bovee, J. V.; McDonnell, L. A.; 
Wuhrer, M. Anal. Chem. 2016, 88, 5904.

[72] Zhou, X. X.; Yang, S.; Yang, G. L.; Tan, Z. Q.; Guan, F. Chin. Chem. Lett. 2019, 30, 676.

[73] Nishikaze, T.; Tsumoto, H.; Sekiya, S.; Iwamoto, S.; Miura, Y.; Tanaka, K. Anal. Chem. 2017, 89, 2353.

[74] Suzuki, N.; Abe, T.; Natsuka, S. Anal. Biochem. 2019, 567, 117.

[75] Furukawa, J. I.; Hanamatsu, H.; Nishikaze, T.; Manya, H.; Miura,
N.; Yagi, H.; Yokota, I.; Akasaka-Manya, K.; Endo, T.; Kanagawa, M.; Iwasaki, N.; Tanaka, K. Anal. Chem. 2020, 92, 14383.

[76] Peng, Y.; Wang, L.; Zhang, Y.; Bao, H.; Lu, H. Anal. Chem. 2019, $91,15993$.

(Cheng, B.) 